\title{
Prints Lubrication, a Factor Influencing the Quality of the Cast Parts
}

\author{
Nicoleta RACHIERU \\ University of Pitesti, Romania, nicoleta.rachieru@upit.ro
}

\begin{abstract}
In today's market economy, where customer demand is increasingly diversified and where value for money is a basic consideration, companies are continuously adapting, increasing their performance and focusing on the use of modern and improved technological processes and equipment to increase profits and reduce losses. To be ideal, the parts produced must meet the requirements imposed by their specific use, according to regulations and dimensional tolerances and in accordance with agreements with the customer. This article deals with aspects of aluminium die-casting, its importance for the technological casting process, the lubrication of the casting mould prints, and the problems that can appear, in terms of casting quality, due to improper pulverization. In a case study carried out on a part of a vehicle component, respectively the Oil bath, a constructive improvement of the Nozzle head is proposed. This improvement led to a reduction in the number of damaged pressure casting parts.
\end{abstract}

\section{Keywords}

die-casting, aluminium, mould fingerprint, spray head, lubrication

\section{Introduction}

In the automotive industry, many components are made of cast aluminium [1]. For example, at Dacia [2] aluminium casting parts are: clutch housing, cap closures, cylinder crankcase, a series of brackets, oil bath, etc. This is a great advantage because it produces raw parts in-house. The weight of these parts varies between 0.3 to $22 \mathrm{~kg}$. The raw material consists of aluminium alloy ingots that are delivered from suppliers in Romania, Poland, Russia and Italy.

The casting process is carried out under pressure using 24 automated presses with the latest technology and capacities ranging from 660 to 2,500 ton-force ( 6.6 to $25 \mathrm{MN}$ ), depending on the type of casting. The casting process is based on the physical principle that any liquid takes the shape of the vessel containing it [3].

For large presses (2,500 tons), the entire technological process is automatic: alloy temperature control, mould thermal regime, casting, part extraction, detachment of the casting network. The parts are controlled in laboratories owned by a quality department, both from a dimensional and material quality point of view.

\section{Casting Cycle}

Obtaining parts, made of aluminium alloys, on die-casting machines means going through a casting cycle. Before starting this cycle, the alloy must be melted and transported to the oven of the casting machine and to the shell prepared for injection (thermal equilibrium). The steps of this cycle are [4]:

1. Mould pulverization, with separator, lubricant and stripping agent and air blowing from the mould, to remove the excess pulverizing agent (which, in contact with the liquid alloy, turns into gas, difficult to remove from hidden areas and which, once accumulated, can favour the appearance of casting imperfections in the part: porosities such as bubbles) (Figure 1).

2. Shell closure. The closing force $\left(F_{i}\right)$ machine (expressed in tons) must take into account the injection force:

$$
F_{i}=F=\frac{P_{s} \times S_{t}}{1000}
$$

where

$F=$ Injection force (piston) [tons];

$F_{i}=$ Closing force (machine) [tons]; 
$P_{s}=$ Specific pressure (applied to the alloy) [bar];

$S_{t}=$ Total projected area $\left[\mathrm{cm}^{2}\right]$.

3. Casting of liquid alloy in the (robotic) injection chamber at a suitable temperature $\left(660-670^{\circ} \mathrm{C}\right)$. The moulding spoon is selected from the existing range according to the amount of liquid alloy required to inject the part (Figure 2).

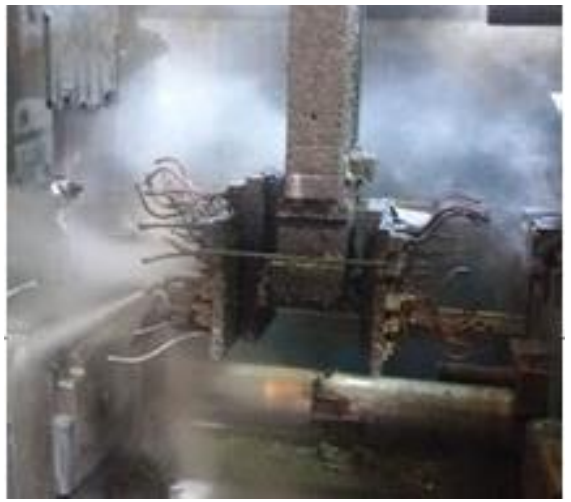

Fig. 1. Mould pulverization

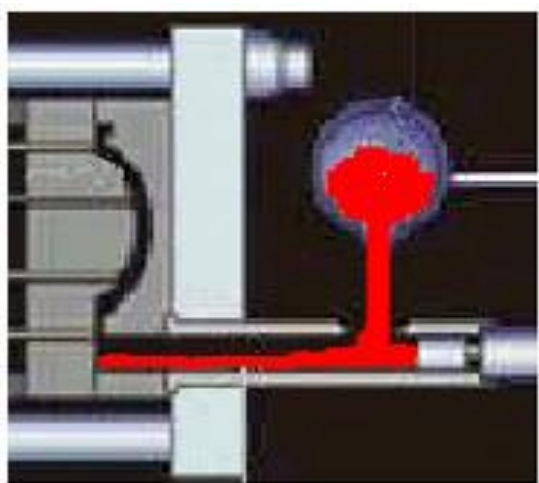

Fig. 2. Alloy casting in the injection chamber [4]

\section{Injection, with the 3 phases:}

Injection phase 1 (F1) = pushing of the alloy, by the injection piston, close to the filling attack sections of the part, at low speed, to avoid the inclusion of air from the injection chamber, in the liquid alloy (found in the part in the form of porosities, such as bubbles).

Injection phase 2 (F2) = filling of the shape (part and surplus of material) in a very short time by increasing the rate of the injection piston forward to avoid solidifying the alloy before filling the shape gap.

Injection phase 3 (F3) = late application after the end of phase 2 of additional pressure on the injection piston, when the alloy is still in a paste-like state, for compacting the part, compensating for shrinkage, taking into account the solidification of the alloy of the difference in density between the liquid state $\left(2.55 \mathrm{~g} / \mathrm{cm}^{3}\right)$ and the solid state $\left(2.75 \mathrm{~g} / \mathrm{cm}^{3}\right)$.

5. Shape solidification alloy (keep the shell closed for a certain time for solidification) (Figure 3).

6. Shell opening (Figure 4).

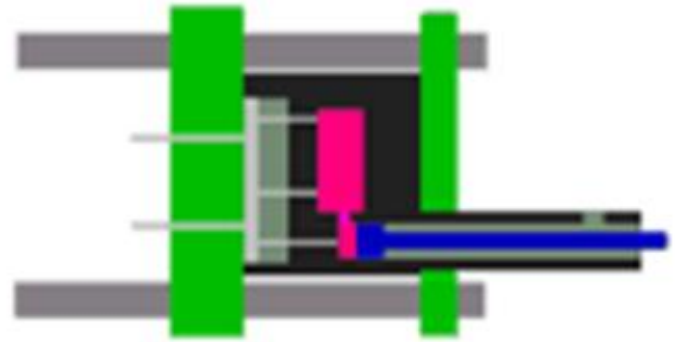

Fig. 3. Solidification alloy

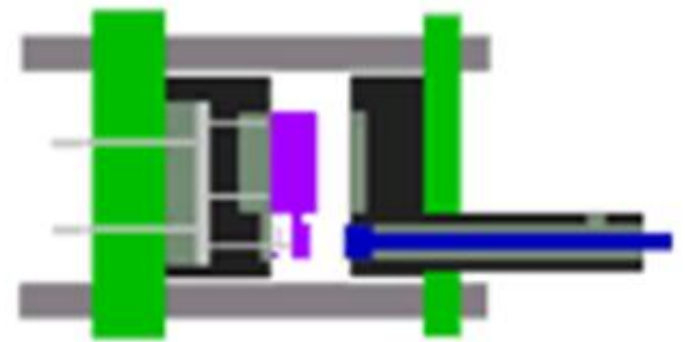

Fig. 4. Shell opening

\section{Opening the mobile cores.}

8. Removal of the shell and extraction (pickup) robotically or manually, in the range $320-250{ }^{\circ} \mathrm{C}$, the contraction coefficient of aluminium alloy, is higher than in steel below $250{ }^{\circ} \mathrm{C}$ and there is a risk of catching (sticking) around the cores, catching pieces or even the whole piece, in shape.

\section{Prints Lubrication and Influence on the Quality of Castings}

The lubrication of the prints, and thus the achievement of thermal equilibrium, is one of the decisive factors in achieving quality in pressure die-casting. Prints (shell) lubrication means the functioning of the creation (formation) of a lubricant film (anti-sticking) and removal on the hot surfaces of the shell. Even if you have a machine with advanced technology and a well known and perfectly executed shell, it is impossible to obtain good quality parts and high production rates without adequate cavity lubrication.

The quantity, type of product, as well as the mode of distribution on the shell prints are important 
factors that are often not analyzed at their true value. Technically appropriate (suitable) lubrication products are not an auxiliary factor at all, they are an essential part of the process in order to achieve the best benefits and performance, Figure 5 .

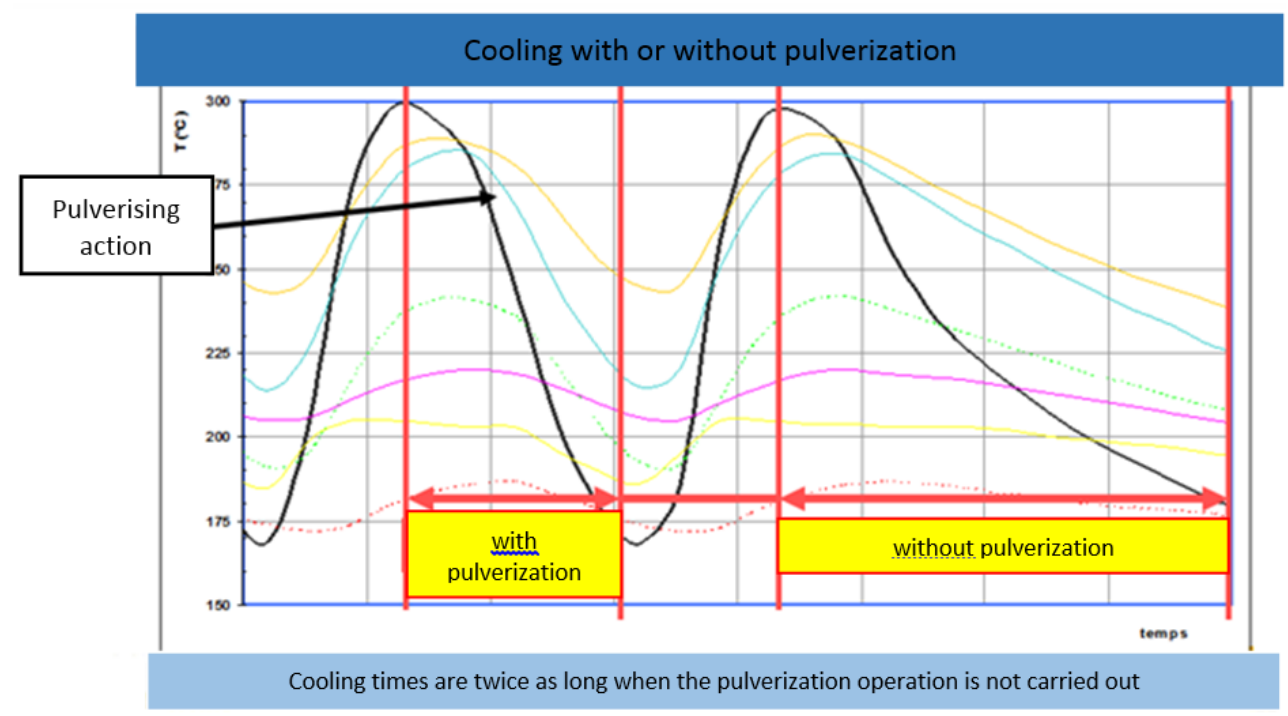

Fig. 5. Cooling with or without pulveriser

Lubricating products must be capable of creating a film at $250-300{ }^{\circ} \mathrm{C}$ and consist of active parts (mineral oils, additives, emulsifiers) and water; a thin film or the absence of it, in various hidden areas or near attacks, inevitably leads to the appearance of adhesions on the shell.

Automatic lubrication ensures consistently good quality, a reduction in the percentage of scrap and an increase in productivity (Tcyc).

The role of preparing the shell for a new injection of the liquid alloy is given by pulverization by:

- decreasing the temperature of the shell (prints) to form the protective film;

- formation of physico-chemical barriers between the shell steel and the liquid alloy;

- reduction of the reactivity of the alloys with respect to the shell surface [5].

\subsection{Defects due to improper pulverization}

The pulverization product, modified by the very large quantity of bacteria, can be a cause of the appearance of defects on the parts.

The product degraded by the bacteria, has lost some of its properties (in particular the granulometry has increased, the emulsion is no longer protective and demulsified), often leading to problems such as: microporosities, welding, deformations during extraction.

The film destroyed and formed due to excessive or low temperature lubrication on the shell leads to the appearance of traces on the part.

Severe (or total) damage to the film in certain areas (such as those close to the attacks) can leave the shell unprotected and, in these cases, it is common to glue or weld between the alloy and the shell, Figure 6. Excessive gas development occurs when the film is destroyed in contact with the liquid alloy.

Other defects and their causes are:

Bumps - incorrectly oriented nozzles, high breath rate, Figure 7;

Voids - heavy pulverization, Figure 8;

Burr - material stuck to the mould, improper pulverization, Figure 9;

Fissure - pulverization, alignment, concentration, pulverisation rate, Figure 10;

Flourish - occur when the first pulverisation are instantly solidified and remain on the workpiece,

Figure 11.

The causes that lead to the formation of the above defects are: initial mould temperature, spray distance, air and product pressure, nozzle output, pulverization time, product concentration, pulverization angle. 


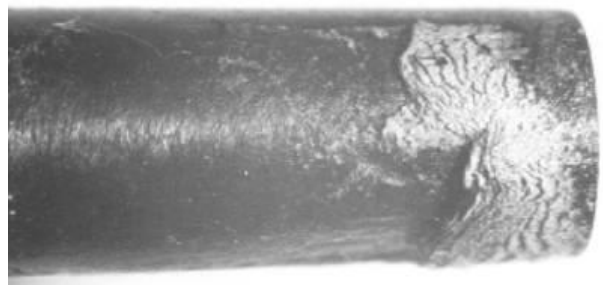

Fig. 6. Brazing

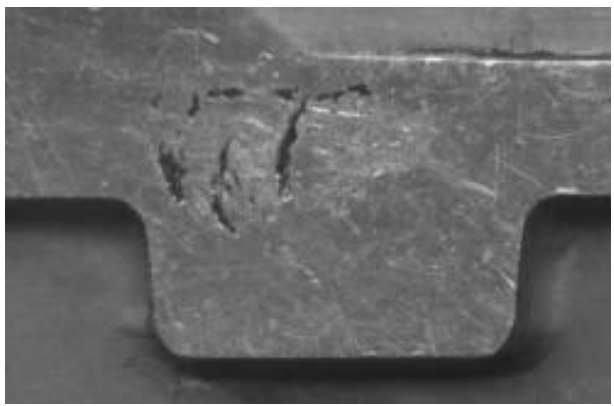

Fig. 8. Voids

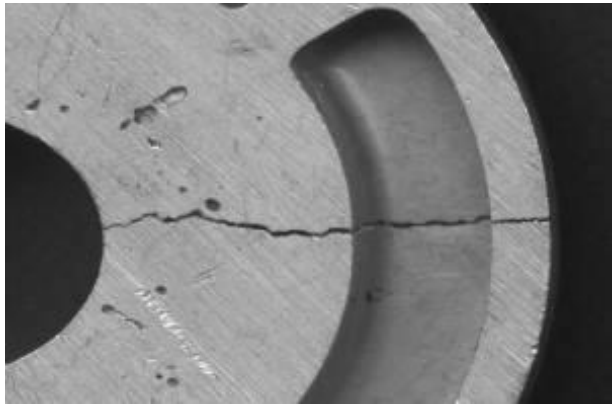

Fig. 10. Fissure

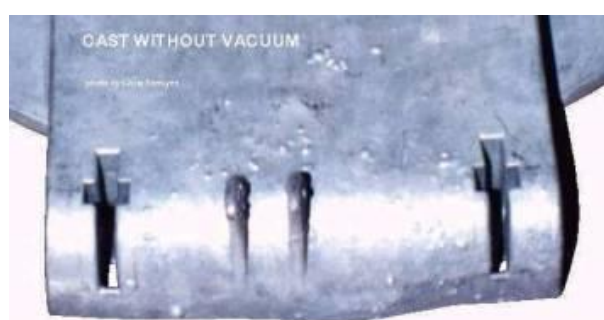

Fig. 7. Bumps

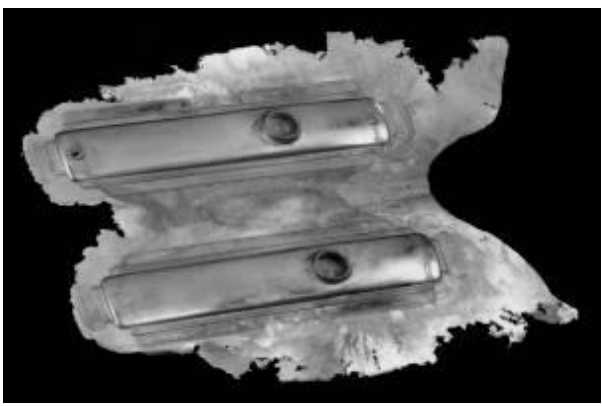

Fig. 9. Burr

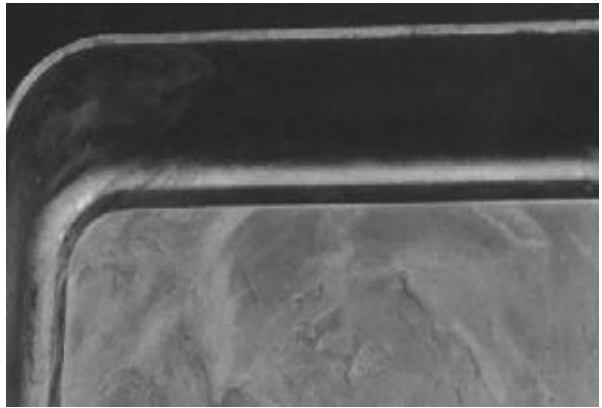

Fig. 11. Flourish

\subsection{Constructive improvement of the PULVERISER HEAD}

Various manufacturers of pulverizing equipment and installations have developed their own designs, but theoretically the principle is the same: a two-arm telescopic assembly allows the handling of a pulverizing head vertically and horizontally, between the two semi-matrics, manually or automatically, computer-assisted maneuvers for automatic memory and reproduction of the trajectory and the pulverizing program.

The pulverizer head, is a modular assembly of product dispensers and air blowing nozzles, mounted in fixed positions, these nozzles being orientable in space within certain technically possible limits. If necessary, when pulverization is deficient in the standard position, the extensions must be used for product nozzles or air nozzles (shaded areas, hidden cores, dents). Unused positions on a standard pulverizer head must be covered by plates designed for this purpose.

The construction of a Pulveriser head, depends on:

- the geometry of the shell to be lubricated;

- thermal circuitry (thermal appearance inside the shell);

- lubricant used;

- requirements required by the shell surface, which must be met by the pulverization and lubrication technique.

Using a universal head for all shells will not improve profitability, even if the parts look similar to achieve this.

Customization of the pulveriser head must address both the very complex lubrication issues and the overall requirements of the benchmark. 


\section{Case Study - Shell Pulverizer Head Upgrade Used in Car Oil Baths \\ 4.1. Current pulverizer head}

The pulverizing head, Figure 12, of the moving part and the fixed part of the oil bath (shown in Figure 13) consists of three sections for the pulverizing solution and one section for blowing out the air. Nozzles and pulverizing pipes are mounted on the spraying sections, and on the air section, the nozzles and air blowing pipes.

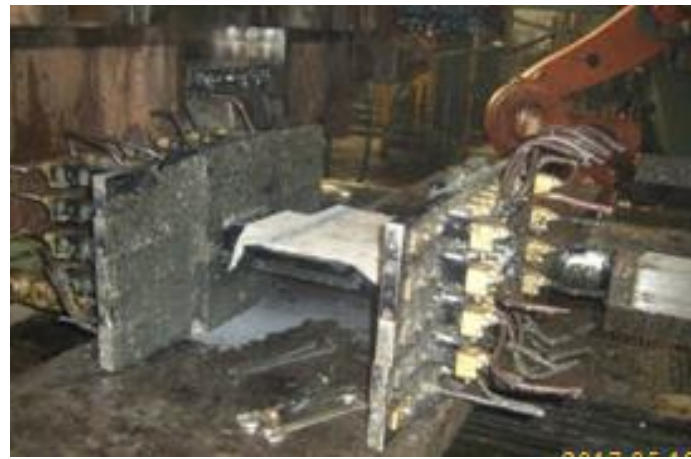

Fig. 12. Pulveriser head [6]

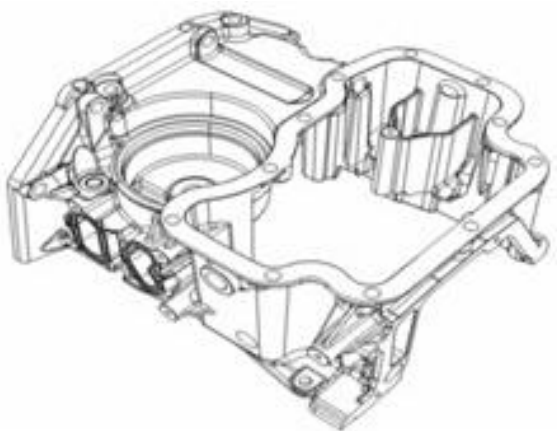

Fig. 13. Oil bath

The nozzles used on the pulverizing head are nozzles with external mixing (low lubricant consumption, small drop size, and better cooling and film formation), in which copper pipes are mounted to facilitate spraying in hard-to-reach areas.

Disadvantages of using this pulverizing head: cumbersome movement of the pulverizing head, clogging of the pipes due to wax deposits, instability of the pipes, corrosion of pipes and nozzles, spot cooling, thermal shock, production of grippe, adhesions, use of a limited number of nozzles.

For the improvement of the pulveriser head were considered:

- the geometry of the shell to be lubricated (impact points);

- thermal circuits (thermal inside the shell, thermography before and after pulverization).

The thermography is intended to check the jet, in order to avoid the appearance of defects on the print (handles).

- the requirements of the shell surface, which must be met by the pulverization and lubrication technique;

- lubricant used.

\subsection{Improved pulverizing head}

Due to the configuration of the prints, the shell has points that are difficult to reach (shadow points in relation to the position and/or direction of the jet) and, generally being very hot spots, require careful and adapted pulverization. Thus, for spraying left/right ports, copper pipes with $90^{\circ}$ angle adapters will be replaced, in the extension of the plates and nozzles will be mounted.

For spraying deeper areas, a few extensions will be mounted on the plates in the head where the distributors will be mounted. Adapter plates can be installed on the upper/lower port-cores, or extensions can be installed at the end of which special distributors can be installed that can pulverize in two directions at the same time (Figure14).

Figure 15 shows a special pulverizing head, made to improve the casting process. It is equipped with additional plates for spraying the ports by placing additional pulverizing nozzles. Thus, by increasing the amount of product pulverized on the mould, we reduce the cycle time per part.

The development of the pulverized head as well as the program, is done at the machine, by repeated casting, adjusting and following the influence of the pulverization, step by step, on the batches of parts, for the following requirements:

- intense pulverization on the hot zones to ensure zonal cooling of the shell and the creation of a lubricating film;

- making the separating film as uniform as possible over the entire surface of the prints (without the appearance of welding); 
- make adequate blower for the removal of waste liquids after pulverization;

- complete extraction of the semi-fabrication from the shell print, which means a good demulsification role for the pulverization solution;

- avoiding deposits in certain areas of the shell by using nozzles adjusted to the flow rate;

- completion of the pulverization-blowing cycle, in the shortest possible time to ensure a high casting rate.

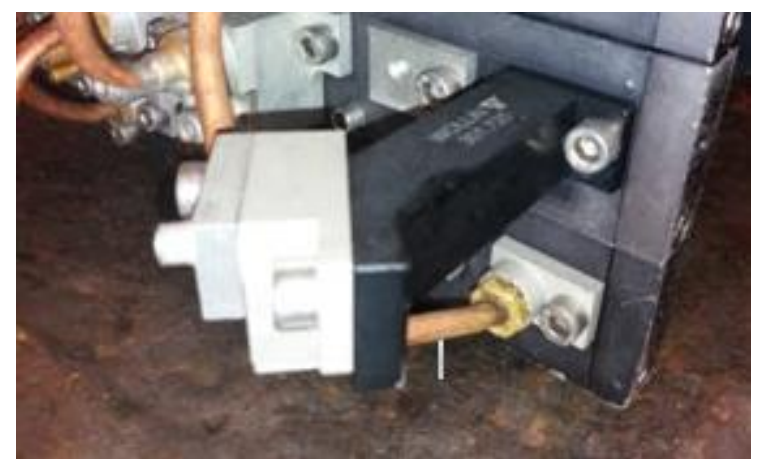

Fig. 14. Extender with special dispenser [6]

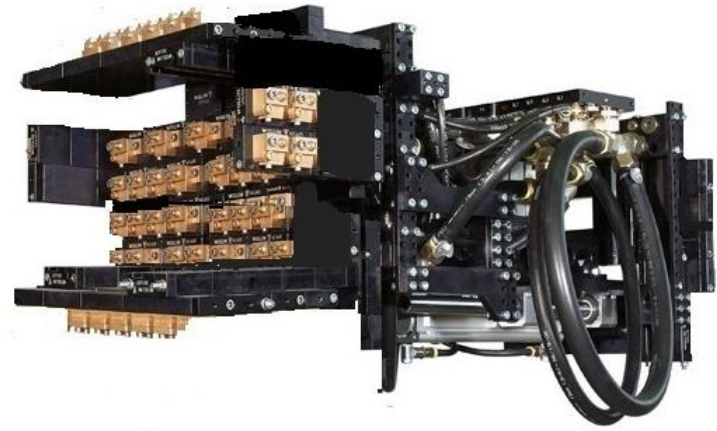

Fig. 15. Special pulveriser head [6]

\section{Conclusion}

Due to the economic production possibilities, by modern casting and processing methods, good corrosion resistance, high thermal conductivity, pleasant appearance and low weight, a number of aluminium parts such as engine blocks, trusses, pistons, cylinders, radiators, gearbox casings, oil baths, body parts and ornaments, rims etc. are made from alloys of this material.

In order to meet the requirements of quality, cost and performance of motor vehicles, the concerns of specialists are oriented towards the practical exploitation of the latest scientific discoveries by developing revolutionary technologies in the field of metallic and ferrous and non-ferrous alloys, the treatment of metals and non-metals by non-conventional processes, the automation and robotization of manufacturing and assembly processes, the design and realization of systems for designing, testing, repairing and controlling computer-assisted production.

The modification of the pulverizing head leads to better handling, shorter cycle time, reduced lubricant consumption, larger coverage area, increased shell life by reducing thermal shocks to the metal. In addition, the percentage of discarded parts was much lower.

\section{References}

1. Buzatu M., Moldovan P. (2009): Elaborarea aliajelor neferoase (Non-ferrous alloys casting). Politehnica Press, University Politehnica of Bucharest, ISBN 978-606-521-091-2, București, Romania (in Romanian)

2. Nițu L. Ed. (coord.) et al. (2013): Procese de fabricaţie specifice industriei de automobile (Manufacturing processes specific to the automotive industry). University of Pitesti Publishing House, ISBN 978-606-560-329-5, Pitești, Romania (in Romanian)

3. Chircor M., Zagan R., Chițu G. (2005): Elemente fundamentale de tehnologia materialelor (Fundamentals of Materials Technology). Editura Ex Ponto, ISBN 973-644-447-3, Constanța, Romania (in Romanian)

4. Banu Il. (2012): Tehnologia Materialelor (Materials technology). University of Pitesti Publishing House, ISBN 978-606-560-291-5, Pitești, Romania (in Romanian)

5. http://www.scritub.com/tehnica-mecanica/particularitati-ale-turnarii-L35412.php. Accesed in 20.05.2020

6. Automobile Dacia S.A. - specific technical documentation 